\title{
Association of caffeine and related analytes with resistance to Parkinson disease among LRRK2 mutation carriers
}

\author{
A metabolomic study
}

Grace F. Crotty, MD, Romeo Maciuca, PhD, Eric A. Macklin, PhD, Junhua Wang, PhD, Manuel Montalban, BS, Sonnet S. Davis, PhD, Jamal I. Alkabsh, BS, Rachit Bakshi, PhD, Xiqun Chen, MD, PhD, Alberto Ascherio, MD, DrPH, Giuseppe Astarita, PhD, Sarah Huntwork-Rodriguez, PhD, and Michael A. Schwarzschild, MD, PhD

Neurology ${ }^{\circledR}$ 2020;95:e3428-e3437. doi:10.1212/WNL.0000000000010863

\section{Abstract}

\section{Objective}

To identify markers of resistance to developing Parkinson disease (PD) among LRRK2 mutation carriers $(L R R K 2+)$, we carried out metabolomic profiling in individuals with PD and unaffected controls (UC), with and without the LRRK2 mutation.

\section{Methods}

Plasma from 368 patients with PD and UC in the LRRK2 Cohort Consortium (LCC), comprising 118 LRRK2+/PD+, 115 LRRK2+/UC, 70 LRRK2-/PD+, and 65 LRRK2-/UC, and CSF available from 68 of them, were analyzed by liquid chromatography with mass spectrometry. For 282 analytes quantified in plasma and CSF, we assessed differences among the 4 groups and interactions between LRRK2 and PD status, using analysis of covariance models adjusted by age, study site cohort, and sex, with $p$ value corrections for multiple comparisons.

\section{Results}

Plasma caffeine concentration was lower in patients with PD vs UC $(p<0.001)$, more so among $L R R K 2+$ carriers (by 76\%) than among LRRK2- participants (by 31\%), with significant interaction between LRRK2 and PD status $(p=0.005)$. Similar results were found for caffeine metabolites (paraxanthine, theophylline, 1-methylxanthine) and a nonxanthine marker of coffee consumption (trigonelline) in plasma, and in the subset of corresponding CSF samples. Dietary caffeine was also lower in $L R R K 2+/ \mathrm{PD}+$ compared to $L R R K 2+/ \mathrm{UC}$ with significant interaction effect with the LRRK2+ mutation $(p<0.001)$.

\section{Conclusions}

Metabolomic analyses of the LCC samples identified caffeine, its demethylation metabolites, and trigonelline as prominent markers of resistance to PD linked to pathogenic LRRK2 mutations, more so than to idiopathic PD. Because these analytes are known both as correlates of coffee consumption and as neuroprotectants in animal PD models, the findings may reflect their avoidance by those predisposed to develop $\mathrm{PD}$ or their protective effects among LRRK2 mutation carriers.

\author{
Correspondence \\ Dr. Crotty \\ grace.crotty@ \\ mgh.harvard.edu
}




\section{Glossary}

A2AR = adenosine 2A receptor; $\mathbf{A C N}=$ acetonitrile; $\mathbf{A N C O V A}=$ analysis of covariance; $\mathbf{B H}=$ Benjamini-Hochberg; IPA = isopropanol; LC/MS = liquid chromatography with mass spectrometry; LCC = LRRK2 Cohort Consortium; LRRK2 = leucinerich repeat kinase 2; mH\&Y = modified Hoehn \& Yahr scale; MJFF = Michael J. Fox Foundation for Parkinson's Research; MoCA = Montreal Cognitive Assessment; OR = odds ratio; PD = Parkinson disease; $\mathbf{U C}=$ unaffected controls.

Leucine-rich repeat kinase 2 (LRRK2) gene mutation is considered a major causative influence on Parkinson disease (PD), and demonstrates variable age-dependent, incomplete penetrance. ${ }^{1,2}$ This incomplete penetrance suggests that other genetic or environmental factors modulate the gene's expression or its effects on PD pathophysiology. The identification of such modulators could pave the way for future preventative and disease-modifying therapies. Our recent work identified higher levels of urate in LRRK2 mutation carriers without PD, suggesting that plasma urate could be a marker of resistance against developing PD in mutation carriers. ${ }^{3}$ Alcalay et al. ${ }^{4}$ studied urinary bis(monoacylglycerol) phosphate isoforms and found slightly higher levels of 2,20-di18:1-bis(monoacylglycerol)phosphate in LRRK2+/PD+ compared to LRRK2+/unaffected controls (UC).

In this study, we carried out metabolomic profiling in participants enrolled in the LRRK2 Cohort Consortium (LCC). To our knowledge, there has only been one plasma metabolomic study of LRRK2 mutation carriers, and its investigation of purine metabolites was limited to $12 L R R K 2+/ \mathrm{PD}+$ participants and 21 LRRK2+/UC. ${ }^{5}$ Our study sought to identify markers of resistance to developing PD in LRRK2 mutation carriers, and to characterize a metabolomic signature of pathogenic LRRK2 mutations. Among our prespecified hypotheses was that caffeine and its related analytes are reduced in patients with PD compared to UC, with reduction similar in those with and without LRRK2 mutations. Our expectation was based on the link between caffeine intake to reduced risk of $\mathrm{PD}^{6-8}$ as well as on the demonstration of their lower serum levels in idiopathic PD. ${ }^{9,10}$

\section{Methods}

\section{Participants}

Plasma and CSF were obtained from participants enrolled in the LCC. The LCC was established in 2009 and has been coordinated and funded by the Michael J. Fox Foundation for Parkinson's Research (MJFF). The LCC includes participants diagnosed with idiopathic PD (LRRK2-/PD+), pathogenic LRRK2 gene mutation carriers with PD (LRRK2+/PD+), $L R R K 2$ gene mutation carriers without evidence of $\mathrm{PD}$ (LRRK2+/UC), and unaffected noncarrier controls (LRRK2-/ UC). The LCC comprises 3 distinct studies, all of which were drawn upon in selecting samples for our research: 23 andMe blood collection study, the LRRK2 longitudinal study, and the LRRK2 cross-sectional study. Participants in the LRRK2 longitudinal study and LRRK2 cross-sectional study were enrolled in family-, community-, or clinic-based studies from North
America, Europe, Asia, and North Africa. The LRRK2 crosssectional study was subdivided into the cross-sectional North American site cohort and the cross-sectional Europe, Asia, and North African site cohort, with their biological samples originally stored in separate biorepositories (Coriell and BioRep, respectively) before all LCC biosamples were transferred for collective management at Indiana University. Therefore, in our study we adjusted analyses for these 4 distinctly processed LCC study site cohorts: the 23andMe blood collection cohort, the LRRK2 longitudinal cohort, the LRRK2 cross-sectional North American cohort, and the LRRK2 cross-sectional Europe, Asia, and North African cohort. Further information on the LCC was published previously ${ }^{11}$ and is available at michaelifox.org/datasets and michaelffox.org/lccinvestigators.

From these 4 LCC study site cohorts, plasma specimens were selected to best match age and sex across the genotype and disease status groups, similar to our prior study. ${ }^{3}$ Sixteen percent of requested plasma specimens had corresponding CSF specimens available. MJFF provided codes linking each LCC biospecimen to the associated participant's genotype, disease status, age, sex, and clinical data including caffeine intake only after our finalized analyte values were submitted to the MJFF LCC depository.

\section{Samples}

The LRRK2 longitudinal study and both of the LRRK2 crosssectional studies had standardized protocols for plasma collection, whereas the 23andMe blood collection study did not (michaeljfox.org/data-sets and 23andme.com/pd/). CSF was collected only in the LRRK2 cross-sectional study (table e1, data available from Dryad, doi:10.5061/dryad.nzs7h44pj). As per protocol, samples were to be collected between 8 and 10 AM with participants strongly advised to be in a fasting state, with a minimum of 8 hours since last meal or food intake. Training videos were provided for collecting, storing, and shipping samples. Further information is provided at files. michaeljfox.org/LRRK2_Cohort_Consortium_Biologics_ Manual\%20_US.pdf. Dietary caffeine questionnaires were completed by participants in the LRRK2 longitudinal study and the LRRK2 cross-sectional study. Using data from the Food and Drug Administration, we converted the cups of caffeinated coffee, black tea, green tea, and soda into milligrams of caffeine per day (fda.gov/consumers/consumerupdates/spilling-beans-how-much-caffeine-too-much).

\section{Standard protocol approvals, registrations, and patient consents}

We did not require ethical approval for this study as it involved anonymized, minimal risk LCC data. 


\section{Sample preparation}

Plasma and CSF samples received from the LCC were stored at $-80^{\circ} \mathrm{C}$ and were thawed for assay on wet ice. Plasma samples $(10 \mu \mathrm{L})$ were spun down and then transferred to polypropylene 96-well V-bottom half deep-well plates. Next, methanol (200 $\mu \mathrm{L})$ containing internal standards was added into each well using the Velocity 11 Bravo Liquid Handling Platform (Agilent Technologies, Santa Clara, CA). Plates were shaken for $5 \mathrm{mi}-$ nutes at room temperature and placed at $-20^{\circ} \mathrm{C}$ for 1 hour to allow further precipitation of proteins. Plates were then centrifuged at $4,000 \mathrm{~g}, 4^{\circ} \mathrm{C}$ for 20 minutes. The supernatant was divided into 2 aliquots: a $100 \mu \mathrm{L}$ aliquot was transferred to 96well plates with glass inserts for direct liquid chromatography with mass spectrometry (LC/MS) analyses of lipids and metabolites (tables e2-e9, data available from Dryad, doi:10. 5061/dryad.nzs7h44pj); a second $50 \mu \mathrm{L}$ aliquot was transferred to a separate 96-well plate with glass inserts, dried down under gentle nitrogen stream, and resuspended in $100 \mu \mathrm{L}$ of $92.5 / 5 /$ 2.5 acetonitrile $(\mathrm{ACN}) /$ isopropanol (IPA)/water with $5 \mathrm{mM}$ ammonium formate and $0.5 \%$ formic acid for LC/MS analysis of GlcCer and GalCer species (tables e2-e9, data available from Dryad, doi:10.5061/dryad.nzs7h44pj).

CSF samples $(20 \mu \mathrm{L})$ were spun down, transferred into 96well plates with glass inserts, then methanol $(100 \mu \mathrm{L})$ containing internal standards was added into each well using the Velocity 11 Bravo Liquid Handling Platform (Agilent Technologies, Santa Clara, CA). Plates were sealed, shaken for 5 minutes at room temperature, and then centrifuged at $1,000 \mathrm{~g}$ at $4^{\circ} \mathrm{C}$ for 2 minutes. Next, the samples were divided into 2 aliquots: a $60 \mu \mathrm{L}$ aliquot was transferred to 96-well plates with glass inserts for direct LC/MS analyses of lipids and metabolites; a $30-\mu \mathrm{L}$ aliquot was transferred to a separate 96-well plate with glass inserts, dried down under gentle nitrogen stream, and resuspended in $30 \mu \mathrm{L}$ of $92.5 / 5 / 2.5$ $\mathrm{ACN} / \mathrm{IPA} /$ water with $5 \mathrm{mM}$ ammonium formate and $0.5 \%$ formic acid for LC/MS analysis of GlcCer and GalCer species (tables e2-e9, data available from Dryad, doi:10.5061/ dryad.nzs7h44pj).

\section{Data reporting}

For each analyte, both un-normalized peak area (units $=$ area) and peak area normalized to an internal standard (units = area ratio) were calculated and reported. For urate, concentration was calculated (units $=\mathrm{mg} / \mathrm{dL}$ ) as a product of the corresponding area ratio and the amount of internal standard used. Peak areas with signal-to-noise $<3$ were not reported. The primary analysis outcome is the internal standard normalized area ratio ("area ratio" or relative abundance).

Figure 1 Volcano plots of plasma metabolomics results
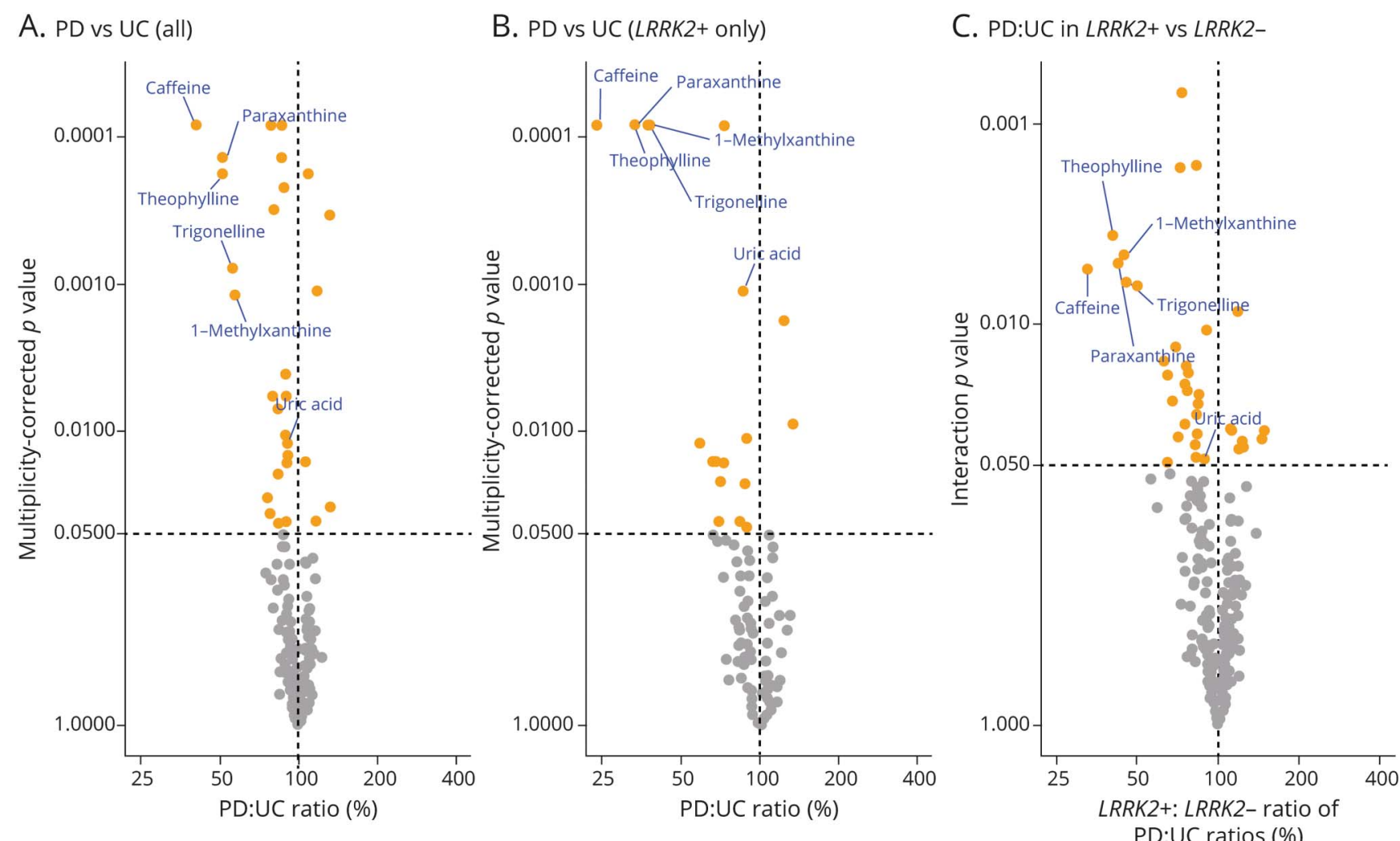

Volcano plots of plasma metabolomics results comparing Parkinson disease (PD): unaffected controls (UC) (ratio for all LRRK2 Cohort Consortium participants analyzed (A), PD:UC ratio for LRRK2+ participants (B), and PD:UC ratio in LRRK2+ vS LRRK2- participants (C). $p$ Values were related to the PD:UC ratio (A and B) or LRRK2+:LRRK2- ratio (C) for each measured analyte adjusted for age, sex, study site cohort, and (in A only) LRRK2 status. 
Table 1 Baseline demographics and features by LRRK2 and Parkinson disease (PD) status of participants contributing analyzed plasma samples

\begin{tabular}{|c|c|c|c|c|c|}
\hline Demographic features & $\begin{array}{l}\text { LRRK2-/UC } \\
(\mathrm{n}=65)\end{array}$ & $\begin{array}{l}\text { LRRK2-/PD+ } \\
(\mathrm{n}=70)\end{array}$ & $\begin{array}{l}\text { LRRK2+/UC } \\
(\mathrm{n}=115)\end{array}$ & $\begin{array}{l}\text { LRRK2+/PD+ } \\
(\mathrm{n}=118)\end{array}$ & $p$ Value $^{a}$ \\
\hline Age, y, mean (SD) & $63.0(9.1)$ & $64.4(8.5)$ & $61.0(8.4)$ & $63.9(8.6)$ & 0.03 \\
\hline Male, n (\%) & $28(43)$ & $38(54)$ & $52(45)$ & $55(47 \%)$ & 0.56 \\
\hline \multicolumn{6}{|l|}{ Study site cohort } \\
\hline LRRK2 longitudinal $(n=87)$ & 19 & 27 & 14 & 27 & \\
\hline LRRK2 cross-sectional North America $(n=68)$ & 21 & 20 & 11 & 16 & $<0.001$ \\
\hline $\begin{array}{l}\text { LRRK2 cross-sectional Europe, Asia, North Africa } \\
\qquad(n=126)\end{array}$ & 25 & 23 & 30 & 48 & \\
\hline 23andMe $(n=87)$ & 0 & 0 & 60 & 27 & \\
\hline Hours fasted, ${ }^{b}$ mean (SD) [n with this info] & $9.5(5.4)[n=63]$ & $8.4(5.5)[n=63]$ & $10.1(5.0)[n=50]$ & $9.7(5.2)[n=85]$ & 0.36 \\
\hline Caucasian race, $\mathrm{n}(\%)$ [n with this info] & $62(95)[n=65]$ & $70(100)[n=70]$ & $55(100)[n=55]$ & $88(97)[n=91]$ & 0.16 \\
\hline MoCA, mean (SD) [n with this info] & $26.4(2.5)[n=64]$ & $26.2(4.4)[n=66]$ & $26.5(2.7)[n=51]$ & $24.5(4.6)[n=76]$ & 0.001 \\
\hline mH\&Y, median (IQR) [n with this info] & $0(0)[n=65]$ & $2(0.5)[n=69]$ & $0(0)[n=54]$ & $2(0.5)[n=88]$ & $<0.001$ \\
\hline $\begin{array}{l}\text { Dietary caffeine intake, mg/d, mean (SD) [n with } \\
\text { this info] }\end{array}$ & $\begin{array}{l}146.6(101.2) \\
{[n=48]}\end{array}$ & $\begin{array}{l}216.2(193.9) \\
{[n=51]}\end{array}$ & $\begin{array}{l}241.6(148.1) \\
{[n=43]}\end{array}$ & $\begin{array}{l}157.4(124.6) \\
{[n=70]}\end{array}$ & 0.002 \\
\hline
\end{tabular}

Abbreviations: IQR = interquartile range; $\mathrm{mH} \& Y=$ modified Hoehn \& Yahr scale; MoCA = Montreal Cognitive Assessment; UC = unaffected controls.

a Analysis of variance for comparison across groups of age, hours, MoCA, and dietary caffeine intake. Kruskal-Wallis for comparison across groups of mH\&Y.

$\chi^{2}$ for comparison across groups of male sex, study site cohort, and Caucasian race.

${ }^{b} \mathrm{~A}$ minimum of 8 hours fasting prior to blood draw between 8 and 10 AM was advised for all participants (except those in the $23 a n d M e$ cohort).

A total of 282 analytes were quantified in the plasma and CSF. We excluded 7 analytes from our PD and UC comparisons, and from our volcano plots (figure 1, A-C), as they correlated closely with antiparkinsonian medication use (i.e., levodopa, its metabolites dopamine, 3-methoxytyrosine, dopamine 3-osulfate, dopamine 4-sulfate, 3-hydroxytyrosol, and the lysine metabolite 3-hydroxy-N6,N6,N6-trimethyllysine.). Plasma urate concentrations measured previously by highperformance liquid chromatography in the same samples ${ }^{3}$ correlated well with those measured by LC/MS in the current study $(r=0.95)$.

Results from 4 of 380 available LCC plasma specimens received for this study did not pass quality control checks and were excluded from further analysis prior to unblinding. Following formal unblinding (but while unaware of individual participants' analyte values) we identified 9 UC classified participants as having features indicative of a PD or related diagnosis (e.g., clinician rating of PD probability of $50 \%-100 \%$, modified Hoehn \& Yahr scale [mH\&Y] scale score $\geq 1$, or Unified Parkinson's Disease Rating Scale part 3 score $\geq 18$ ), suggesting initial misclassification of their enrollment status. After review of these cases with their site clinicians and study cohort staff (and while unaware of any analyte values participants), with concurrence of MJFF staff, we corrected the classification status of 1 participant and excluded from analysis the remaining 8 participants. Of note, these LCC participant classification corrections were made since our previously published analysis of the dataset. ${ }^{3}$

\section{Statistical analysis}

Sample size $(\mathrm{n}=380$, with $\mathrm{n}=120$ for each of LRRK2+/PD+ and $L R R K 2+/ \mathrm{UC}$, the 2 critical comparison groups) was determined based on estimation of sample size required to achieve adequate power for replication of a urate difference between these groups in the original test sample analysis $(\mathrm{n}=64$ for each of $L R R K 2+/ \mathrm{PD}+$ and $L R R K 2+/ \mathrm{UC}$ groups; $p=0.047$ ).

A statistical analysis plan was specified prior to unblinding of investigators to LRRK2 genotype, PD status, and all other clinical parameters linked to plasma and CSF samples. For plasma and CSF metabolites, we reported the geometric mean of relative abundance. For all plasma analytes, estimates of mean differences between PD and UC participants and between LRRK2+ and LRRK2- participants were generated with a robust analysis of covariance (ANCOVA) model with $\log 2$ (area ratio) as the dependent variable, and adjusting by PD status cohort (PD vs UC), LRRK2 gene status, sex, age (modeled as cubic spline, with a knot at 60 years), and substudy (the 4 study site cohorts). Interaction terms of PD status cohort $\times L R R K 2$ status $\times$ sex and PD status cohort $\times$ substudy were included. The ANCOVA model was the basis for pairwise tests for the contrasts of interest (PD vs UC, overall or within LRRK2 \pm subgroups), associated $p$ values, and adjusted between-groups effect sizes. To partially control 
Figure 2 Chemical structures and metabolic pathways of caffeine and related analytes (paraxanthine, theophylline, 1methylxanthine, and trigonelline)

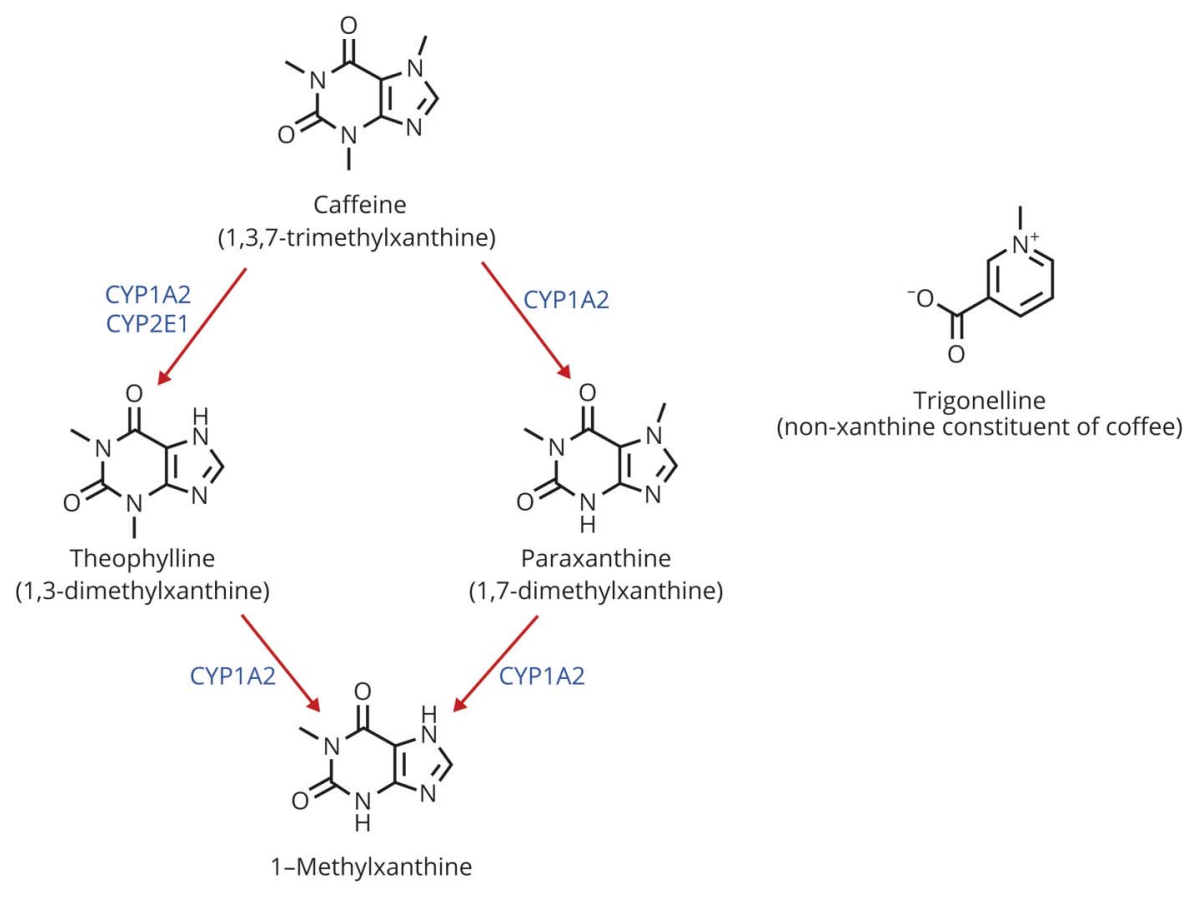

the false discovery rate, we employed a Benjamini-Hochberg $(\mathrm{BH})$ correction to the $p$ values for the PD vs UC (overall, and within each of the LRRK2+ and LRRK2- groups) comparisons. Statistical significance was judged at a 2 -sided 0.05 significance level.

For the smaller subset of participants with CSF samples, between-group estimates were generated from a joint CSF plasma linear mixed effects model, considering the CSF/ plasma measurements as repeated, within-participant measures for each analyte, adjusting for the same set of covariates as above. A similar, albeit simplified set of interaction terms was included in the model (cohort $\times$ LRRK2 status $\times$ sample type, sex $\times$ sample type, and age $\times$ sample type).

\section{Data availability}

Raw data from this study are available from online LCC data request at michaeljfox.org/data-sets. Supplemental data (tables e1-e9, doi:10.5061/dryad.nzs7h44pj) are stored on Dryad.

\section{Results}

Table 1 presents baseline characteristics of the 368 LCC participants whose plasma was analyzed for this study, stratified by PD status (1:1 for diagnosed PD:UC) and LRRK2 gene status (2:1 for LRRK2+:LRRK2-). Differences among the 4 groups' features highlight the value in adjusting for age, sex, and study site cohort. As expected, Montreal Cognitive Assessment (MoCA) scores were lower in the PD groups compared to UC $(p<0.05)$. Data on time fasted prior to blood draw and on race, MoCA, and mH\&Y assessments were available for most participants except for the $24 \%$ from the 23andMe blood collection study (which provided plasma only from $L R R K 2+$ individuals). Regarding specific pathogenic LRRK2 variants present, 91\% $(\mathrm{n}=107)$ of the LRRK2+/PD+ and 92\% ( $\mathrm{n}=106) L R R K 2+/ \mathrm{UC}$ groups carried the G2019S LRRK2 mutation. Other LRRK2 variants in the LRRK2+/PD+ group were R1441G ( $=4), \mathrm{R} 1441 \mathrm{C}$ $(\mathrm{n}=1), \mathrm{N} 1437 \mathrm{H}(\mathrm{n}=2), \operatorname{L1795F}(\mathrm{n}=2), \mathrm{C} 228 \mathrm{~S}(\mathrm{n}=1)$, and unknown $(\mathrm{n}=1)$. Other LRRK2 variants in the $L R R K 2+/ \mathrm{UC}$ group were R1441G ( $\mathrm{n}=4), \mathrm{R} 1441 \mathrm{C}(\mathrm{n}=1), \mathrm{N} 1437 \mathrm{H}(\mathrm{n}=$ $1)$, L1114L $(n=1)$, and L1795F $(n=2)$.

Comparing the concentrations of 282 LC/MS-quantified plasma metabolites between people with and without PD irrespective of LRRK2 gene status revealed a cluster of 5 analytes showing the greatest differences, excluding metabolites linked to antiparkinsonian medication. All 5 are caffeinerelated analytes (figure 2) and are lower in the participants with PD than UC, as depicted in figure 1A in a volcano plot of metabolomics data with effect size (ratio for PD vs UC values, adjusted for age, sex, study site cohort, and LRRK2 status) plotted against $\mathrm{BH}$-adjusted $p$ values. Compared to UC, those with PD had lower plasma levels not only of caffeine (by $71 \%$ ) but also of its partially demethylated xanthine-based metabolites paraxanthine (1,7-dimethylxanthine), theophylline (1,3-dimethylxanthine), 1-methylxanthine (by $57 \%, 56 \%$, and $49 \%$, respectively), and trigonelline, a non-xanthine constituent of coffee (by 52\%), with associated $\mathrm{BH}$-adjusted $p$ values all $\leq 0.001$ (table 2). Consistent with their metabolic and 
Table 2 Caffeine, its metabolites, and trigonelline in plasma of LRRK2 Cohort Consortium participants by LRRK2 and Parkinson disease (PD) status with adjusted geometric mean concentrations (95\% confidence interval)

\begin{tabular}{lllllll}
\hline Plasma analyte & $\begin{array}{l}\text { LRRK2-/UC } \\
(\mathrm{n}=65)\end{array}$ & $\begin{array}{l}\text { LRRK2-/PD+ } \\
(\mathrm{n}=\mathbf{7 0})\end{array}$ & $\begin{array}{l}\text { LRRK2+/UC } \\
(\mathrm{n}=\mathbf{1 1 5})\end{array}$ & $\begin{array}{l}\text { LRRK2+/PD+ } \\
(\mathrm{n}=\mathbf{1 1 8})\end{array}$ & $\begin{array}{l}\text { BH-adjusted } p \text { value, } \\
\text { for PD vs UC }\end{array}$ & $\begin{array}{l}\text { Interaction } p \text { value, PD:UC } \\
\text { for LRRK2+ vs }-\end{array}$ \\
\hline Caffeine & $9.7(6.1-15.5)$ & $6.7(4.2-10.9)$ & $12.2(7.9-18.7)$ & $2.9(2.0-4.2)$ & $<0.001$ & 0.005 \\
\hline Paraxanthine & $2.9(2.1-4.1)$ & $2.3(1.6-3.3)$ & $3.7(2.7-5.1)$ & $1.2(0.9-1.6)$ & $<0.001$ & 0.005 \\
\hline Theophylline & $1.7(1.2-2.4)$ & $1.3(0.9-1.9)$ & $2.1(1.5-3.0)$ & $0.7(0.5-0.9)$ & $<0.001$ & 0.004 \\
\hline 1-Methylxanthine & $\begin{array}{l}0.0007 \\
(0.0005-0.001)\end{array}$ & $\begin{array}{l}0.0006 \\
(0.0004-0.0008)\end{array}$ & $\begin{array}{l}0.001 \\
(0.0008-0.001)\end{array}$ & $\begin{array}{l}0.0004 \\
(0.0003-0.0005)\end{array}$ & 0.001 & 0.005 \\
\hline Trigonelline & $1.3(0.9-1.8)$ & $1.1(0.8-1.5)$ & $1.9(1.4-2.6)$ & $0.7(0.5-0.9)$ & $<0.001$ & 0.006 \\
\hline
\end{tabular}

Abbreviations: $\mathrm{BH}=$ Benjamini-Hochberg; $\mathrm{UC}=$ unaffected controls.

dietary relationships, plasma caffeine correlated well with its demethylation products (paraxanthine, theophylline, and 1-methylxanthine) and its coffee co-constituent, trigonelline, with Spearman coefficients $(r)$ of approximately $0.8,0.8,0.5$, and 0.5 , respectively, in participants with PD.

Stratifying by genotype reveals that the extent to which these caffeine-related analytes are lower in PD is substantially greater among pathogenic LRRK2 mutation carriers (figure $1, \mathrm{~B}$ and C, and table 2). In $L R R K 2+/ P D+$ plasma, caffeine itself was $76 \%$ lower than in plasma of $L R R K 2+/ \mathrm{UC}$ participants, whereas in idiopathic PD plasma caffeine was 31\% lower than in LRRK2-/ UC participants ( $p$ for $L R R K 2 \times$ PD status interaction $=0.005$ ). Similarly, levels in PD were even lower among LRRK2+ vs LRRK2 - participants for paraxanthine (66\% vs $21 \%)$, theophylline $(67 \%$ vs $21 \%)$, 1-methylxanthine ( $62 \%$ vs $14 \%)$, and trigonelline ( $63 \%$ vs $15 \%$ ), with $p$ for interaction $<0.01$ for each. Of note, fasting times prior to blood draw did not differ appreciably across groups (table 1), suggesting that the lower levels of analytes from caffeinated beverages observed in the plasma of people with PD are not due to their having fasted longer.

Dietary caffeine consumption questionnaire data were available for 212 of the participants with plasma caffeine metabolites (table 1). In this subset, participants with PD with the LRRK2 mutation consumed significantly (41\%) less caffeine (in $\mathrm{mg} / \mathrm{d}$ ) when compared to UC with the LRRK2 mutation $(p<0.003)$, and there was a significant interaction effect with the LRRK2 mutation $(p<0.001)$. Dietary caffeine consumption positively correlated with plasma caffeine metabolite concentration $(r=0.3)$. When caffeine metabolite concentration was adjusted for dietary caffeine intake, the significant difference between PD and controls $(p=$ $0.01)$ and between LRRK2+/PD+ and LRRK2+/UC ( $p=$ 0.01 ) persisted, although the interaction effect for $L R R K 2$ mutation was not significant $(p=0.38)$.

Similar to plasma caffeine, plasma levels of the endogenous purine urate were found to be a marker of $\mathrm{PD}$ resistance among LRRK2 mutation carriers in our earlier, nonmetabolomic analysis in the nearly identical LCC sample set. ${ }^{3}$ Although urate also inversely associates with PD among LRRK2+ more than LRRK2 - participants, the associations are weaker than for the caffeine-related analytes ${ }^{3}$ and the evidence for an interaction between urate, LRRK2 genotype, and PD status reached only marginal statistical significance in our more recent analysis $(p=0.047)$ (figure 1, A-C).

Matching CSF samples were available for 68 participants with analyzed plasma: LRRK2-/UC $(\mathrm{n}=14), L R R K 2-/ \mathrm{PD}+(\mathrm{n}=$ 18), LRRK2+/UC ( $=18)$, and LRRK2+/PD+ ( $=18)$, all of whom were from the LRRK2 cross-sectional study (table e-1, data available from Dryad, doi:10.5061/dryad.nzs7h44pj). In figure 3, we present boxplot illustrations of the overlapping plasma and CSF samples of the 4 groups by LRRK2 gene status and PD status. Among the LRRK2 mutation carriers in this subset, both CSF and plasma caffeine levels were significantly lower in participants with PD than in UC (after adjustment for age, sex, and study site cohort): by $74 \%$ in CSF $(p<0.02)$ and by $76 \%$ in plasma $(p=0.01)$, consistent with a strong positive correlation between the entire sample of plasma and CSF caffeine concentrations $(r=0.90)$. By contrast, among LRRK2 - participants in this subset, CSF caffeine was $23 \%$ lower and plasma caffeine was $24 \%$ higher in participants with PD compared to controls ( $p=0.65$ and 0.71 , respectively).

Similar associations were observed for CSF levels of caffeine's dimethyl metabolites paraxanthine and theophylline, and of trigonelline, whose plasma and CSF levels also closely correlated ( $r=0.94,0.94$, and 0.90, respectively). 1-methylxanthine was not measured in the CSF. Among the LRRK2 mutation carriers in this subset, both CSF and plasma levels of paraxanthine, theophylline, and trigonelline were lower in participants with PD than in UC after adjustment: by $73 \%, 74 \%$, and $70 \%$ for CSF $(p=0.003,0.003$, and $<0.001)$ and by $74 \%, 76 \%$, and $69 \%$ for plasma $(p=0.002,<0.002$, and 0.001$)$, respectively. By contrast, among LRRK2- participants in this subset, levels of paraxanthine, theophylline, and trigonelline were largely indistinguishable after adjustment between those with and without PD, being higher in CSF by $2 \%, 1 \%$, and $9 \%$ 

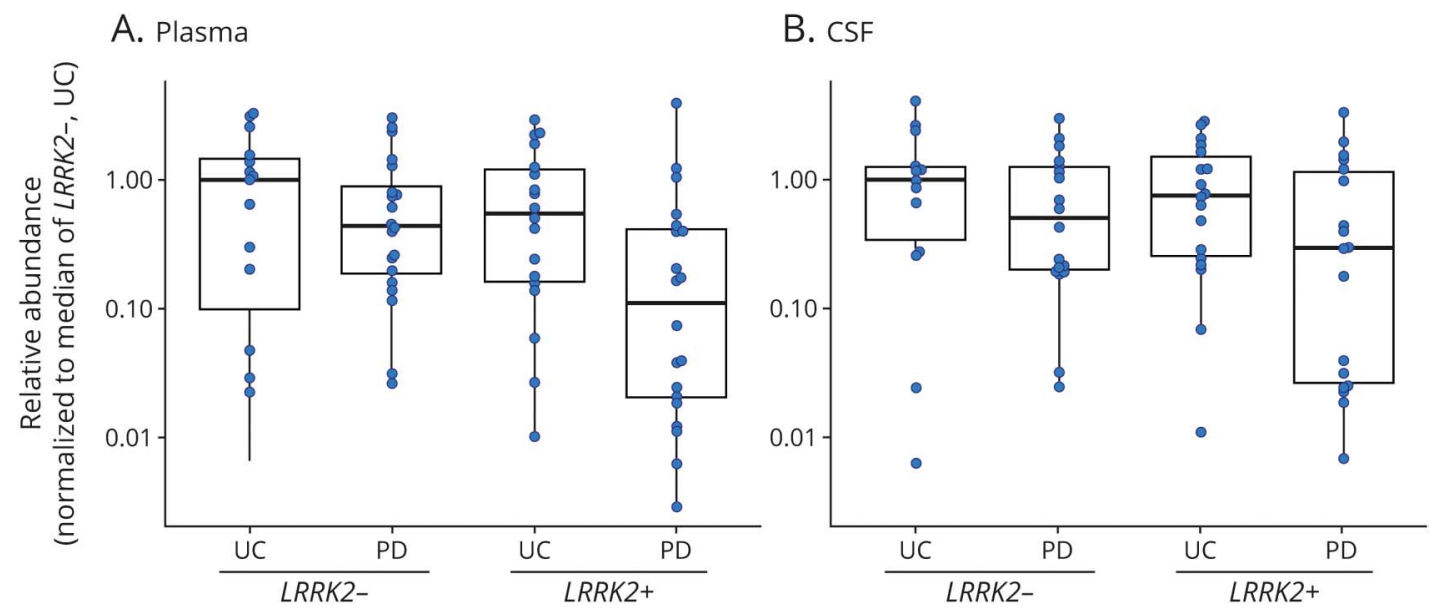

Concentrations of caffeine in matched plasma (A) and CSF (B) samples of LCC participants by LRRK2 and Parkinson disease (PD) status, adjusted for age, sex, and study site cohort. UC = unaffected controls.

in those with vs without PD ( $p=0.97,0.99$, and 0.82$)$, and in plasma by $20 \%, 19 \%$, and $37 \%$ in those with vs without PD $(p=$ $0.70,0.72$ and 0.40$)$. Fasting times prior to lumbar puncture, like those for blood collection, did not differ across the 4 groups at approximately 12 hours $(p=0.29)$.

Exploratory stratification showed that the low caffeine association with $\mathrm{PD}$ among $L R R K 2$ mutation carriers was present irrespective of sex (62\% lower with PD among men and 85\% lower with PD among women; $p=0.004$ and $<0.001$, respectively) or age ( $65 \%$ lower with PD among those younger than 60 years at baseline, and $83 \%$ lower among those 60 years or older at baseline; $p<0.02$ and $<0.001$, respectively). Similarly, lower caffeine with PD among LRRK2 mutation carriers was just as strong when PD cases were restricted to the $22 \%$ who appear not to have been taking levodopa (based on its plasma metabolite 3-methoxytyrosine having relative abundance levels less than 0.1 ), being $78 \%$ lower with PD $(p<0.001)$, or to those at an early clinical stage ( $\mathrm{mH} \& \mathrm{Y}$ 2.5 or lower), being $76 \%$ lower with PD $(p=0.002)$, suggesting that the lower caffeine levels with PD among LRRK2 mutation carriers is not likely due to an antiparkinsonian medication effect or more advanced disease. In contrast to LRRK2 mutation carriers, those without mutations (LRRK2-) showed a nonsignificant, weaker link between low caffeine and PD, with caffeine $39 \%$ lower in men $(p=0.25)$ and $21 \%$ in women $(p=0.56)$.

\section{Discussion}

In this metabolomics study of LRRK2 mutation carriers, we identified the dietary purine caffeine and its metabolites as the most affected pathway in plasma with significantly lower levels of caffeine, its demethylation products, and trigonelline, a nonpurine marker of coffee consumption in participants with $\mathrm{PD}$ compared to UC, and to a significantly greater extent among pathogenic LRRK2 mutation carriers than among those without a mutation.
Our observation of lower plasma caffeine concentrations in participants with PD confirms recent metabolic ${ }^{9}$ and metabolomic $^{10}$ findings, consistent with well-established epidemiologic evidence for increased risk of developing idiopathic PD in individuals who consume fewer caffeinated beverages, based on dietary recall. ${ }^{12}$ Interestingly, Fujimaki et al. ${ }^{9}$ did not find significantly lower caffeine intake in participants with PD despite lower serum levels of caffeine and its metabolites in their cohort, raising the possibility that the lower levels could result from reduced bioavailability (e.g., due to reduced gastrointestinal absorption). By contrast, in the larger LCC cohort assessed here, a lower intake among PD vs control participants, and a significantly lower intake in participants with $L R R K 2+/ \mathrm{PD}+$ compared to LRRK2+/UC, suggest that lower concentrations of caffeine and its metabolites circulating in people with PD reflects, at least in part, their lesser consumption of this dietary purine. Of note, the finding of comparably reduced plasma levels of trigonelline, a nonpurine constituent and plasma biomarker of coffee ${ }^{13}$ (which comprised $84 \%$ of the total daily caffeine consumption), further supports a dietary basis for the lower caffeine concentrations in PD vs controls. However, our results are also consistent with a role of differential absorption, metabolism, or clearance of caffeine in PD given the persistent, significantly lower level of caffeine and its related analytes in our participants with PD with or without a pathogenic LRRK2 mutation, compared to their control counterparts, following adjustment for dietary caffeine intake.

The unexpected finding that caffeine's and related analytes' associations with resistance to PD are substantially greater among LRRK2 mutation carriers than noncarriers appears robust and intriguing. Levels of these analytes were lower in both the plasma and CSF of participants with PD vs UC among LRRK2 mutation carriers, whereas among noncarriers these analytes were not significantly reduced in patients with PD vs control participants. Direct evidence for interaction between PD and LRRK2 status $(p<0.01$ for each of the 5 
caffeine-related analytes) suggests a true gene-environment interaction, rather than sample size bias resulting from the (2: 1) predominance of $L R R K 2+$ over $L R R K 2-$ participants in this study's LCC sampling.

Corroborating these metabolomic data, we obtained complementary epidemiologic evidence that participants with PD consumed significantly less caffeine compared to UC only among LRRK2 mutation carriers. Interestingly, our findings of LRRK2-caffeine intake interaction substantiate earlier findings of Kumar et al. ${ }^{14}$ They studied the interplay between caffeine consumption and a different LRRK2 mutation-the R1628P risk variant in a Chinese population. They similarly reported a significant LRRK2-caffeine interaction, with a lower odds ratio (OR) of developing PD among R1628P LRRK2 mutation carriers who consumed caffeine (OR 3.1) than among mutation carriers who were nonconsumers (OR 15.4) when compared to caffeine consumers without the mutation, ${ }^{14}$ suggesting that the biology underlying the LRRK2-caffeine interaction entails the common ability of COR domain-based R1628P and kinase domain-based G2019S mutations to increase kinase activity. ${ }^{15-17}$

Similar but indirect evidence for caffeine's greater association with resistance to genetic forms of PD was recently provided by Angelopoulou et al., ${ }^{18}$ who explored environmental factors in early-onset (before 50 years of age) compared to lateronset PD in a Greek cohort. They observed that coffee drinking was associated with a lower risk of early but not lateronset PD. They also detected a linear dose association between coffee exposure and the risk of developing familial as well as early-onset PD. ${ }^{18}$ As early-onset PD and familial PD are more likely to be genetic than later-onset $\mathrm{PD}$, these data together with those of Kumar et al. ${ }^{14}$ and ours suggest that caffeine could be broadly associated with PD gene penetrance.

Of note, no statistically significant analyte-LRRK2 mutation interaction has been reported previously for PD risk at the metabolome level. Urate - an endogenous antioxidant and end product of purine metabolism - was found in our preceding study of the nearly identical LCC cohort to be associated with PD among LRRK2 mutation carriers to a greater extent than in noncarriers. ${ }^{3}$ However, in contrast to caffeine's link to PD resistance, that of urate was relatively modest and only slightly greater among $L R R K 2$ mutation carriers than noncarriers (figure 1, A-C).

Our findings for CSF levels of caffeine and related analytes were similar to those for their plasma counterparts, despite the lower number of CSF $(n=68)$ than plasma $(n=368)$ samples analyzed. The similarities are in keeping with the close correlation between plasma and CSF concentrations for these analytes. These findings support the use of peripheral (e.g., plasma) samples in assessing the caffeine metabolic pathway in relation to PD risk.

Caffeine is the most widely consumed psychoactive substance and a nonselective antagonist of the adenosine $2 \mathrm{~A}$ receptor (A2AR). It also possesses neuroprotective properties in animal models of PD.
Both its psychostimulant actions and protective effects on dopaminergic neurons likely rely on A2ARs, ${ }^{19-21}$ with A2AR blockade by caffeine reducing excitotoxic and inflammatory processes. ${ }^{22-24}$ The association of caffeine with resistance to PD could reflect the ability of PD determinants to reduce the likelihood of caffeine intake, or conversely it could reflect a causal relationship driven by a protective effect of caffeine or a related analyte. ${ }^{23,25,26}$ Interestingly, LRRK2 biology may potentiate either of these caffeine-PD relationships given its involvement in striatal neuroplasticity and nigrostriatal neurodegeneration, both of which can be attenuated by caffeine via its actions on adenosine A2ARs. ${ }^{27,28}$ For example, the pathogenic G2019S LRRK2 mutation has been found to alter synaptic plasticity in the A2AR-laden striatum while bolstering resistance to social stress in young animals. ${ }^{29}$ Alternatively, the well-established neuroprotective properties of caffeine or dimethyl metabolites, paraxanthine and theophylline, which are themselves A2AR antagonists ${ }^{30,31}$ in PD models, could be potentiated in the setting of pathogenic LRRK2 mutations. For example, the increased kinase activity of pathogenic G2019S LRRK2 may potentiate the dopaminergic degeneration induced by $\alpha$-synuclein, ${ }^{32}$ and $\alpha$-synuclein-induced dopaminergic neuron injury can be attenuated by caffeine ${ }^{33}$ and depend upon the A2 $\mathrm{AR}^{34}$ Thus, A2AR antagonists like caffeine and its metabolites may be particularly effective in attenuating LRRK2 kinasepotentiated $\alpha$-synuclein pathobiology, raising the possibility that the therapeutic potential of caffeine or other A2AR antagonists may be greater for slowing or preventing LRRK2-PD than for idiopathic disease. Similarly, although the nonpurine trigonelline may simply be a marker of coffee and caffeine consumption, it has been shown to have its own protective effects in a PD model. ${ }^{35}$

There are several strengths to our study. First, our metabolomic analysis of LRRK2-PD was conducted on the largest cohort to date. Second, the cohort included matched LRRK2 gene status as well as PD controls, allowing the opportunity to gauge interactions across genotype and disease state. Third, the depth of the LCC biorepository allowed us to assess CSF as well as plasma biomarkers of LRRK2-PD. Finally, we were able to correlate dietary intake with metabolomics, which is relatively novel in allowing us to explore the basis for lower caffeinerelated analytes in $L R R K 2-P D$.

Several limitations of our study should be noted, including potential selection bias as participants were recruited through the multiple individual study site cohorts that comprise the LCC. Although we endeavored to match or adjust for relevant covariates to reduce their influence when differing across groups, unmeasured confounders could have affected our results. Second, misclassification of PD or control participants could not be fully excluded in part based on the lack of a biomarker for definitive diagnosis of PD, although extensive phenotype data were available for most LCC participants, allowing us to cross-check and confirm classifications, and such errors would have biased us toward null results. Lastly, the results are cross-sectional, precluding direct assessment of the predictive potential of analytes on PD risk and progression. 
The identification of caffeine and adenosine antagonists as potential markers of PD resistance among LRRK2 mutation carriers supports their potential for development as biomarkers contributing to phenoconversion risk assessment among carriers, and to progression rates among participants with LRRK2+ PD. In addition, identification of caffeinerelated analytes as resistance markers raises the possibility of their development as candidate therapeutics given the relatively low risk of repurposing these dietary or pharmacologic agents—caffeine, theophylline, and trigonelline-for slowing progression in those with manifest LRRK2+ PD, or among atrisk mutation carriers to reduce the penetrance of the disease. Next steps may include replication of these metabolomic results in an independent cohort of LRRK2 mutation carriers and assessing their specificity in other genetic (e.g., GBA mutation-driven) forms of PD.

\section{Acknowledgment}

This study was funded by the Michael J. Fox Foundation for Parkinson's Research (to M.A.S.), the Farmer Family Foundation Initiative for Parkinson's Disease Research (to M.A.S.), a Jane \& Alan Batkin Research Fellowship (to G.C. and R.B.), The Edmond J. Safra Fellowship in Movement Disorders (G.C.), and NIH R01NS110879 (to M.A.S.). Data and biospecimens used in preparation of this article were obtained from the Michael J. Fox Foundation-sponsored LRRK2 Cohort Consortium (LCC) under LCC project IDs 108 (plasma) and 122 (CSF). The LRRK2 Cohort Consortium is coordinated and funded by The Michael J. Fox Foundation for Parkinson's Research. The authors thank the participants, investigators, and staff of the LCC; Katherine Callahan for editorial assistance; and Eoghan Hynes for technical support.

\section{Study funding}

The Michael J. Fox Foundation for Parkinson's Research (to M.A.S.), the Farmer Family Foundation Initiative for Parkinson's Disease Research (to M.A.S.), a Jane \& Alan Batkin Research Fellowship (to G.C. and R.B.), The Edmond J. Safra Fellowship in Movement Disorders (to G.C.), and NIH R01NS110879 (to M.A.S.).

\section{Disclosure}

G.F. Crotty reports no disclosures. R. Maciuca is a salaried employee of Denali Therapeutics Inc. E.A. Macklin reports no disclosures. J. Wang is a salaried employee of Denali Therapeutics Inc. M. Montalban is a salaried employee of Denali Therapeutics Inc. S. Davis is a salaried employee of Denali Therapeutics Inc. J.I. Alkabsh is a salaried employee of Denali Therapeutics Inc. R. Bakshi, X. Chen, and A. Alberto report no disclosures. G. Astarita is a salaried employee of Denali Therapeutics Inc. S. Huntwork-Rodriguez is a salaried employee of Denali Therapeutics Inc. M.A. Schwarzschild reports no disclosures. Go to Neurology.org/N for full disclosures.

\section{Publication history}

Received by Neurology May 30, 2020. Accepted in final form August 17, 2020.
Appendix Authors

\begin{tabular}{|c|c|c|}
\hline Name & Location & Contribution \\
\hline $\begin{array}{l}\text { Grace F. Crotty, } \\
\text { MD }\end{array}$ & $\begin{array}{l}\text { Department of } \\
\text { Neurology, } \\
\text { Massachusetts General } \\
\text { Hospital; Harvard } \\
\text { Medical School, Boston }\end{array}$ & $\begin{array}{l}\text { Design and } \\
\text { conceptualization of the } \\
\text { study, major role in the } \\
\text { acquisition of data, } \\
\text { analysis and } \\
\text { interpretation of the data, } \\
\text { drafting and revising the } \\
\text { manuscript for } \\
\text { intellectual content }\end{array}$ \\
\hline
\end{tabular}

\begin{tabular}{ll}
\hline Romeo & Denali Therapeutics Inc., \\
Maciuca, PhD & San Francisco, CA
\end{tabular}

Design and conceptualization of the study, major role in the acquisition of data, analysis and interpretation of the data, revising the manuscript for intellectual content

\begin{tabular}{lll}
\hline Eric A. Macklin, & $\begin{array}{l}\text { Harvard Medical School; } \\
\text { Biostatistics Center, } \\
\text { Department of Medicine, } \\
\text { Massachusetts General } \\
\text { Hospital, Boston }\end{array}$ & $\begin{array}{l}\text { Design and } \\
\text { conceptualization of the } \\
\text { study, major role in the } \\
\text { acquisition of data, } \\
\text { analysis and } \\
\text { interpretation of the data, } \\
\text { revising the manuscript } \\
\text { for intellectual content }\end{array}$ \\
\end{tabular}

Junhua Wang,
PhD
San Francisco, CA

Major role in the acquisition of data, analysis and interpretation of the data, revising the manuscript for intellectual content

\begin{tabular}{lll}
\hline Manuel & Denali Therapeutics Inc., & Major role in the \\
Montalban, BS & San Francisco, CA & acquisition of data, \\
& analysis and \\
& interpretation of the data, \\
& revising the manuscript \\
& for intellectual \\
& content
\end{tabular}

\begin{tabular}{lll}
\hline $\begin{array}{l}\text { Sonnet S. } \\
\text { Davis, PhD }\end{array}$ & $\begin{array}{l}\text { Denali Therapeutics Inc., } \\
\text { San Francisco, CA }\end{array}$ & $\begin{array}{l}\text { Major role in the } \\
\text { acquisition of data, } \\
\text { analysis and } \\
\text { interpretation of the data, } \\
\text { revising the manuscript } \\
\text { for intellectual content }\end{array}$ \\
\hline Jamal & Denali Therapeutics Inc., & $\begin{array}{l}\text { Major role in the } \\
\text { acquisition of data, } \\
\text { alkabsh, BS }\end{array}$ \\
& San Francisco, CA & $\begin{array}{l}\text { anterpretation of the data, } \\
\text { revising the manuscript } \\
\text { for intellectual } \\
\text { content }\end{array}$ \\
& &
\end{tabular}

\begin{tabular}{lll}
\hline $\begin{array}{l}\text { Rachit Bakshi, } \\
\text { PhD }\end{array}$ & $\begin{array}{l}\text { Department of } \\
\text { Neurology, } \\
\text { Massachusetts General } \\
\text { Hospital; Harvard } \\
\text { Medical School, Boston }\end{array}$ & $\begin{array}{l}\text { Design and } \\
\text { conceptualization of the } \\
\text { study, major role in the } \\
\text { acquisition of data, } \\
\text { analysis and } \\
\text { interpretation of the data, } \\
\text { revising the manuscript } \\
\text { for intellectual content }\end{array}$ \\
\hline $\begin{array}{l}\text { Xiqun Chen, } \\
\text { MD, PhD }\end{array}$ & $\begin{array}{l}\text { Department of } \\
\text { Neurology, } \\
\text { Massachusetts General } \\
\text { Hospital; Harvard } \\
\text { Medical School, Boston }\end{array}$ & $\begin{array}{l}\text { Design and } \\
\text { conceptualization of the } \\
\text { study, major role in the } \\
\text { acquisition of data, } \\
\text { analysis and } \\
\text { interpretation of the data, } \\
\text { revising the manuscript } \\
\text { for intellectual content }\end{array}$ \\
\hline
\end{tabular}


Appendix (continued)

\begin{tabular}{ll}
\hline Name & Location \\
\hline Alberto & Harvard Medical School; \\
Ascherio MD & Department of Nutrition, \\
DrPH & Harvard T.H. Chan School \\
& of Public Health, Boston, \\
& MA
\end{tabular}

Contribution

Design and

conceptualization of the

study, major role in the

acquisition of data,

analysis and

interpretation of the data,

revising the manuscript

for intellectual content

\begin{tabular}{ll}
\hline Giuseppe & Denali Therapeutics Inc., \\
Astarita, PhD & San Francisco, CA
\end{tabular}

Design and

conceptualization of the study, major role in the acquisition of data, analysis and interpretation of the data, revising the manuscript for intellectual content

\begin{tabular}{ll}
\hline Sarah & Denali Therapeutics Inc., \\
Huntwork- & San Francisco, CA \\
Rodriguez, PhD &
\end{tabular}

\section{Design and}

conceptualization of the study, major role in the acquisition of data, analysis and interpretation of the data, revising the manuscript for intellectual content

\begin{tabular}{|c|c|c|}
\hline $\begin{array}{l}\text { Michael A. } \\
\text { Schwarzschild, } \\
\text { MD, PhD }\end{array}$ & $\begin{array}{l}\text { Department of } \\
\text { Neurology, } \\
\text { Massachusetts General } \\
\text { Hospital; Harvard } \\
\text { Medical School, Boston }\end{array}$ & $\begin{array}{l}\text { Design and } \\
\text { conceptualization of the } \\
\text { study, major role in the } \\
\text { acquisition of data, } \\
\text { analysis and } \\
\text { interpretation of the data, } \\
\text { drafting and revising the } \\
\text { manuscript for } \\
\text { intellectual content }\end{array}$ \\
\hline
\end{tabular}

\section{References}

1. Healy DG, Falchi M, O'Sullivan SS, et al. Phenotype, genotype, and worldwide genetic penetrance of LRRK2-associated Parkinson's disease: a case-control study. Lancet Neurol 2008;7:583-590.

2. Lee AJ, Wang Y, Alcalay RN, et al. Penetrance estimate of LRRK2 p.G2019S mutation in individuals of non-Ashkenazi Jewish ancestry. Mov Disord 2017;32:1432-1438.

3. Bakshi R, Macklin EA, Logan R, et al. Higher urate in LRRK2 mutation carriers resistant to Parkinson disease. Ann Neurol 2019;85:593-599.

4. Alcalay RN, Hsieh F, Tengstrand E, et al. Higher urine bis(monoacylglycerol)phosphate levels in LRRK2 G2019S mutation carriers: implications for therapeutic development. Mov Disord 2020;35:134-141

5. Johansen KK, Wang L, Aasly JO, et al. Metabolomic profiling in LRRK2-related Parkinson's disease. PLoS One 2009;4:e7551.

6. Ascherio A, Zhang SM, Hernán MA, et al. Prospective study of caffeine consumption and risk of Parkinson's disease in men and women. Ann Neurol 2001;50:56-63.

7. Ascherio A, Schwarzschild MA. The epidemiology of Parkinson's disease: risk factors and prevention. Lancet Neurol 2016;15:1257-1272.

8. Ross GW, Abbott RD, Petrovitch H, et al. Association of coffee and caffeine intake with the risk of Parkinson disease. JAMA 2000;283:2674-2679.

9. Fujimaki M, Saiki S, Li Y, et al. Serum caffeine and metabolites are reliable biomarkers of early Parkinson disease. Neurology 2018;90:e404-e411.
10. Hatano T, Saiki S, Okuzumi A, et al. Identification of novel biomarkers for Parkinson's disease by metabolomic technologies. J Neurol Neurosurg Psychiatry 2016;87: 295-301.

11. Marras C, Alcalay RN, Caspell-Garcia C, et al. Motor and nonmotor heterogeneity of LRRK2-related and idiopathic Parkinson's disease. Mov Disord 2016;31:1192-1202.

12. Costa J, Lunet N, Santos C, et al. Caffeine exposure and the risk of Parkinson's disease: a systematic review and meta-analysis of observational studies. J Alzheimers Dis 2010; 20(suppl 1):S221-S238.

13. Midttun Ø, Ulvik A, Nygård O, Ueland PM. Performance of plasma trigonelline as a marke of coffee consumption in an epidemiologic setting. Am J Clin Nutr 2018;107:941-947.

14. Kumar PM, Paing SST, Li H, et al. Differential effect of caffeine intake in subjects with genetic susceptibility to Parkinson's disease. Sci Rep 2015;5:15492.

15. Berwick DC, Heaton GR, Azeggagh S, Harvey K. LRRK2 biology from structure to dysfunction: research progresses, but the themes remain the same. Mol Neurodegener 2019;14:49.

16. Shu Y, Ming J, Zhang P, et al. Parkinson-related LRRK2 mutation R1628P enables Cdk5 phosphorylation of LRRK2 and upregulates its kinase activity. PLoS One 2016; 11:e0149739.

17. Tan EK, Peng R, Teo YY, et al. Multiple LRRK2 variants modulate risk of Parkinson disease: a Chinese multicenter study. Hum Mutat 2010;31:561-568.

18. Angelopoulou E, Bozi M, Simitsi AM, et al. The relationship between environmental factors and different Parkinson's disease subtypes in Greece: data analysis of the Hellenic Biobank of Parkinson's disease. Parkinsonism Relat Disord 2019;67:105-112.

19. Chen JF, Xu K, Petzer JP, et al. Neuroprotection by caffeine and $\mathrm{A}(2 \mathrm{~A})$ adenosine receptor inactivation in a model of Parkinson's disease. J Neurosci 2001;21:RC143.

20. Lazarus M, Shen HY, Cherasse Y, et al. Arousal effect of caffeine depends on adenosine A2A receptors in the shell of the nucleus accumbens. J Neurosci 2011;31: 10067-10075.

21. Xu K, Di Luca DG, Orrú M, et al. Neuroprotection by caffeine in the MPTP model of Parkinson's disease and its dependence on adenosine A2A receptors. Neuroscience 2016;322:129-137.

22. Bagga P, Chugani AN, Patel AB. Neuroprotective effects of caffeine in MPTP model of Parkinson's disease: a (13)C NMR study. Neurochem Int 2016;92:25-34.

23. Morelli M, Carta AR, Kachroo A, Schwarzschild MA. Pathophysiological roles for purines: adenosine, caffeine and urate. Prog Brain Res 2010;183:183-208.

24. Sonsalla PK, Wong LY, Harris SL, et al. Delayed caffeine treatment prevents nigra dopamine neuron loss in a progressive rat model of Parkinson's disease. Exp Neurol 2012;234:482-487.

25. Schwarzschild MA, Chen JF, Ascherio A. Caffeinated clues and the promise of adenosine A(2A) antagonists in PD. Neurology 2002;58:1154-1160.

26. Xu K, Bastia E, Schwarzschild M. Therapeutic potential of adenosine A(2A) receptor antagonists in Parkinson's disease. Pharmacol Ther 2005;105:267-310.

27. Chen JF, Sonsalla PK, Pedata F, et al. Adenosine A2A receptors and brain injury: broad spectrum of neuroprotection, multifaceted actions and "fine tuning" modulation. Prog Neurobiol 2007;83:310-331.

28. Ferré S, Díaz-Ríos M, Salamone JD, Prediger RD. New developments on the adenosine mechanisms of the central effects of caffeine and their implications for neuropsychiatric disorders. J Caffeine Adenosine Res 2018;8:121-131.

29. Matikainen-Ankney BA, Kezunovic N, Menard C, et al. Parkinson's disease-linked LRRK2-g2019S mutation alters synaptic plasticity and promotes resilience to chronic social stress in young adulthood. J Neurosci 2018;38:9700-9711.

30. Schwarzschild MA, Agnati L, Fuxe K, et al. Targeting adenosine A2A receptors in Parkinson's disease. Trends Neurosci 2006;29:647-654.

31. Xu K, Xu YH, Chen JF, Schwarzschild MA. Neuroprotection by caffeine: time course and role of its metabolites in the MPTP model of Parkinson's disease. Neuroscience 2010;167:475-481.

32. Daher JPL, Abdelmotilib HA, Hu X, et al. Leucine-rich repeat kinase 2 (LRRK2) pharmacological inhibition abates $\alpha$-synuclein gene-induced neurodegeneration. J Biol Chem 2015;290:19433-19444.

33. Yan R, Zhang J, Park HJ, et al. Synergistic neuroprotection by coffee components eicosanoyl-5-hydroxytryptamide and caffeine in models of Parkinson's disease and DLB. Proc Natl Acad Sci USA 2018;115:E12053-E12062.

34. Kachroo A, Schwarzschild MA. Adenosine A2A receptor gene disruption protects in an $\alpha$-synuclein model of Parkinson's disease. Ann Neurol 2012;71:278-282.

35. Gaur V, Bodhankar SL, Mohan V, Thakurdesai PA. Neurobehavioral assessment of hydroalcoholic extract of Trigonella foenum-graecum seeds in rodent models of Parkinson's disease. Pharm Biol 2013;51:550-557. 


\section{Neurology}

\section{Association of caffeine and related analytes with resistance to Parkinson disease among LRRK2 mutation carriers: A metabolomic study}

Grace F. Crotty, Romeo Maciuca, Eric A. Macklin, et al.

Neurology 2020;95;e3428-e3437 Published Online before print September 30, 2020

DOI 10.1212/WNL.000000000010863

This information is current as of September 30, 2020

Updated Information \& Services

References

Citations

Subspecialty Collections

Permissions \& Licensing

Reprints including high resolution figures, can be found at: http://n.neurology.org/content/95/24/e3428.full

This article cites 35 articles, 8 of which you can access for free at: http://n.neurology.org/content/95/24/e3428.full\#ref-list-1

This article has been cited by 1 HighWire-hosted articles: http://n.neurology.org/content/95/24/e3428.full\#\#otherarticles

This article, along with others on similar topics, appears in the following collection(s):

Case control studies

http://n.neurology.org/cgi/collection/case_control_studies

Parkinson's disease/Parkinsonism

http://n.neurology.org/cgi/collection/parkinsons_disease_parkinsonism

Information about reproducing this article in parts (figures,tables) or in its entirety can be found online at:

http://www.neurology.org/about/about_the_journal\#permissions

Information about ordering reprints can be found online:

http://n.neurology.org/subscribers/advertise

Neurology ${ }^{\circledR}$ is the official journal of the American Academy of Neurology. Published continuously since 1951, it is now a weekly with 48 issues per year. Copyright Copyright ( 2020 The Author(s). Published by Wolters Kluwer Health, Inc. on behalf of the American Academy of Neurology.. All rights reserved. Print ISSN: 0028-3878. Online ISSN: 1526-632X.

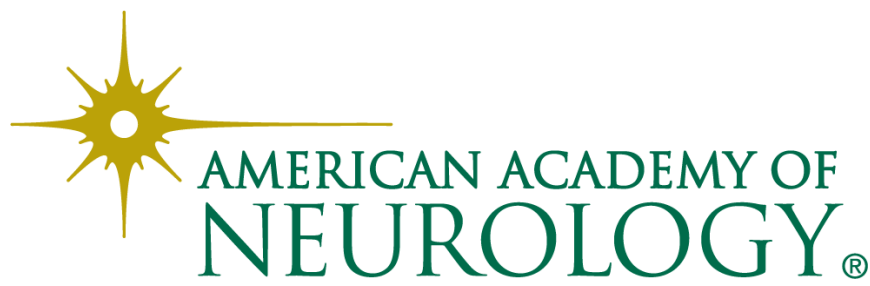

